DIVISION OF THE HUMANITIES AND SOCIAL SCIENCES

CALIFORNIA INSTITUTE OF TECHNOLOGY

PASADENA, CALIFORNIA 91125

A CHARACTERIZATION OF STRATEGIC COMPLEMENTARITIES

Federico Echenique

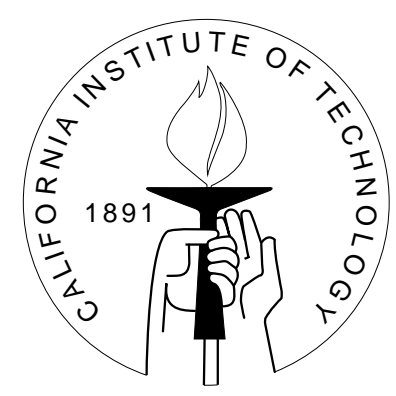

SOCIAL SCIENCE WORKING PAPER 1142

October 2002 


\title{
A Characterization of Strategic Complementarities
}

\author{
Federico Echenique
}

\begin{abstract}
I characterize games for which there is an order on strategies such that the game has strategic complementarities. I prove that, with some qualifications, games with a unique equilibrium have complementarities if and only if Cournot best-response dynamics has no cycles; and that all games with multiple equilibria have complementarities.

As applications of my results, I show: 1. That generic $2 X 2$ games either have no pure-strategy equilibria, or have complementarities. 2. That generic two-player finite ordinal potential games have complementarities.
\end{abstract}

JEL classification numbers: C62, C72 


\title{
A Characterization of Strategic Complementarities
}

\author{
Federico Echenique*
}

\section{Introduction}

A game has strategic complementarities (Topkis 1979, Vives 1990) if, given an order on players' strategies, an increase in one player's strategy makes the other players want to increase their strategies. For example, the players could be firms in price competition; if each firm's optimal price is an increasing function of the prices set by their opponents, the game has strategic complementarities. In games of strategic complementarities (GSC), Nash equilibria have a certain order structure; in particular, there is a smallest and largest equilibrium (Topkis, Vives, Zhou (1994)). Further, the set of all rationalizable strategies, and the set of limits of adaptive learning, is bounded below by the smallest equilibrium and above by the largest equilibrium (Vives 1990, Milgrom and Roberts 1990, Milgrom and Shannon 1994). GSC are a well-behaved class of games, and a useful tool for economists.

Consider the coordination game in Figure 1, is it a GSC? That is, is there an order on players' strategies so that best-responses are monotone increasing? Yes, let $\alpha$ be smaller than $\beta$. Then, player 2's best response to $\alpha$ is $\alpha$ and to $\beta$ is $\beta$. So, when 1 increases her strategy from $\alpha$ to $\beta$, 2's best response increases from $\alpha$ to $\beta$. Similarly for 1 . So, with this order the coordination game is a GSC.

The question I address is: what does this depend on? That is, when can we find an order on strategies so that a game is a GSC? Note that how strategies are ordered is not

*I thank an associate editor and a referee for their comments. I also thank Elvio Accinelli, Bob Anderson, Juan Dubra, Paul Milgrom, Stephen Morris, Charles Púgh, Ilya Segal, Chris Shannon, Xavier Vives, and seminar participants at Arizona State and Stanford Universities. A conversation with Ted O'Donoghue and Clara Wang prompted me to work on the research presented here. The non-standard proof of Theorem 3 owes a great deal to Bob Anderson, I am deeply grateful for his help. I worked out the results in Section 8 in response to Stephen Morris's very stimulating questions. Finally, part of this paper was written while I visited UC Berkeley's Economics Department, I appreciate Berkeley's hospitality. Any errors are my responsibility. 


\begin{tabular}{c|c|c|}
\multicolumn{1}{c}{} & \multicolumn{1}{c}{$\alpha$} & \multicolumn{1}{c}{$\beta$} \\
\cline { 2 - 3 }$\alpha$ & 2,2 & 0,0 \\
\cline { 2 - 3 }$\beta$ & 0,0 & 1,1 \\
\hline
\end{tabular}

Figure 1: A coordination game.

part of the description of a game - it does not affect the available strategies or payoffs. We as analysts use the order as a tool, therefore we are justified in choosing the order to conform to our theory. Often, results on GSC are used by coming up with a clever order on strategies that makes a situation into a GSC, this paper is concerned with how often such orders exists.

We normally introduce strategic complementarities by assuming supermodular payoffs. The crucial feature for most results on GSC is that the game's best-response correspondence is monotone increasing, and the assumption of supermodular payoffs is sufficient for monotone increasing best-responses. ${ }^{1}$ I shall argue that it is enough, for the purpose of this paper, to define a GSC as a game for which there is a partial order on strategies so that best-responses are monotone increasing (and such that strategies have a lattice structure).

My results are:

1. With some qualifications, a game with a unique pure-strategy Nash equilibrium is a GSC if and only if Cournot best-response dynamics has no cycles except for the equilibrium.

2. A game with two or more pure-strategy Nash equilibria is always a GSC.

I illustrate my results with two applications: Generically, $2 X 2$ games are either GSC or have no pure-strategy equilibria. And, generically, a finite two-player ordinal potential game is a GSC, but ordinal potential games with more than two players need not be GSC.

I now discuss my results.

1. With some qualifications, in games with a unique Nash equilibrium, strategic complementarities is equivalent to the absence of cycles in Cournot best-response dynamics. If $b$ is the game's best-response function (the product of the players' best-response

\footnotetext{
${ }^{1}$ In fact, the first paper using lattice-programming techniques in economics, Vives (1985), defined GSC like I do here.
} 
functions) then Cournot best-response dynamics starting at $x$ is defined by $x_{0}=x$, $x_{n}=b\left(x_{n-1}\right), n=1,2, \ldots$. Absence of cycles means that, if $x$ is not an equilibrium, Cournot best-response dynamics starting at $x$ never returns to $x$, i.e. $x_{n} \neq x$ for all $n \geq 1$, or, equivalently, that $x \neq b^{n}(x)$ for all $n \geq 1$. In finite games, absence of cycles is equivalent to global stability, that is that $b^{n}(x)$ converges to the equilibrium for all $x$. In infinite games, absence of cycles is a weaker condition than global stability - so I show that a game with a unique, globally stable, equilibrium is a GSC.

2. A game with two or more pure-strategy equilibria is a GSC, so the vast majority of games that we encounter in applied work are GSC. The order in the coordination game of Figure 1 that makes its best-response function increasing involves making one Nash equilibrium the smallest point in the joint strategy space, and the other equilibrium the largest point in the strategy space. I show that, if a game has at least two equilibria, the same trick always works; we can order the strategies such that one equilibrium is the largest strategy profile and the other equilibrium is the smallest, and such that best-responses are monotone increasing. This result has implications for the use of complementarities to obtain predictions in games.

The literature on GSC has developed a set-valued prediction concept: the set of strategies that are larger than the smallest Nash equilibrium and smaller than the largest Nash equilibrium. This "interval prediction" contains all rationalizable strategies, and all strategies that are limits of adaptive learning. Is the interval prediction in general a sharp prediction? Milgrom and Roberts suggest that the answer may be negative:

Indeed, for some games, these bounds are so wide that our result is of little help: it is even possible that these bounds are so wide that the minimum and maximum elements of the strategy space are equilibria. (Milgrom and Roberts 1990, p. 1258)

Milgrom and Roberts go on to argue that, in some models, "the bounds are quite narrow." They present as examples an arms-race game, and a class of Bertrand oligopoly models, where there is a unique equilibrium.

My results imply that this is generally the case: if a game does not have a unique equilibrium, the interval prediction is essentially vacuous, as all games with multiple equilibria are GSC where the smallest and largest equilibria are the smallest and largest strategy profiles. This happens because games with multiple equilibria always involve a 
kind of coordination problem - namely the problem of coordinating on one equilibriumand this coordination problem can be formalized through an order on strategies that makes the game a GSC.

I show that, in some games, no order that makes the game a GSC avoids a trivial interval prediction. In fact, the coordination game in Figure 1 is such a game. On the other hand, in the coordination game, all strategy profiles are rationalizable, which may suggest that the interval prediction coincides in general with rationalizability. I present an example where no order that makes the game a GSC, avoids strictly dominated strategies in the interval prediction - this is further evidence that the interval prediction is problematic.

I wish to emphasize that GSC are still a very useful tool. In a given game, complementarities jointly with the rest of the structure of the game can provide sharp results. ${ }^{2}$ In a sense, complementarities is like compactness - most spaces we work with can be compactified, but compactness is still a very powerful tool. For example, Topkis's (1979) algorithm for finding the smallest and largest Nash equilibria in GSC is very useful on a given game with complementarities; and once Topkis's algorithm has delivered an interval prediction we can judge if it is sharp or not in that particular game. Other examples are Amir's (1996) elegant methods for analyzing Cournot oligopoly models using complementarities, and the equilibrium uniqueness results in the literature on global games (Morris and Shin 2000). ${ }^{3}$

My results are a contribution to the theory of GSC, delineating the boundaries of the set of GSC, but I do not provide new positive results on GSC, and I do not increase the scope of applicability of existing results on GSC. In fact, applying my results requires a previous knowledge of the equilibrium set of a game that one seldom has in applicationsrather, one tries to use results on GSC to obtain that knowledge. The point of this paper is that complementarities alone do not possess important predictive power, but that we can use complementarities to understand properties of a particular game.

Lippman, Mamer, and McCardle (1987), Sobel (1988), Milgrom and Roberts (1990),

\footnotetext{
${ }^{2}$ Possibly, that is why "natural" orders - such as the usual order on $\mathbf{R}^{n}$-give sharp results: there is a relation between complementarities and the rest of the structure of the game.

${ }^{3}$ Amir's analysis does not proceed by introducing a convenient order on strategy spaces like I do (see also Amir and Lambson (2000)). My results - which, unlike Amir's, require prior knowledge of some equilibria of the game -imply that there are more Cournot oligopoly models that can be made into a GSC than the ones identified by Amir. But the structure, in addition to complementarities, that Amir uses in his paper enables him to obtain substantive results about Cournot oligopoly, and his results cannot be generalized using my characterization.
} 
Milgrom and Shannon (1994), Milgrom and Roberts (1994), Echenique (2002), and Echenique and Sabarwal (2000) present comparative statics results for a parameterized GSC. They prove that, if a parameter is complementary to players' choices, some selections of equilibria are monotone increasing in the parameter. My results prompt the question: can all systematic comparative statics conclusion be rationalized as coming from a parameterized GSC? The answer, it turns out, is no: there are parameterized models (with multiple equilibria) such that no order delivers the comparative statics as an application of results for GSC. In a sense, comparative statics acts as an "identifying restriction," we do not have enough degrees of freedom in selecting the order on strategies so as to make the game a GSC and, at the same time, preserve monotone comparative statics.

In Section 2 I give some preliminary definitions and present the model that the results apply to. Section 3 presents my results for games with a unique equilibrium, and section 4 presents my results for games with multiple equilibria. Section 5 discusses the interval prediction. Section 6 discusses comparative statics restrictions. In section 7 I present an application to $2 X 2$ games, and in section 8 an application to potential games. Section 9 contains the proofs of Theorems 3 and 7 .

\section{Definitions}

\subsection{Preliminaries}

A detailed discussion of the concepts defined in this subsection can be found in Topkis (1998). A pair $(X, \leq)$, where $X$ is a set and $\leq$ is a transitive, reflexive, antisymmetric binary relation, is a partially ordered set; $(X, \leq)$ is totally ordered if, for all $x, y \in X$, $x \leq y$ or $y \leq x(\leq$ is then a total order on $X) ;(X, \leq)$ is a lattice if whenever $x, y \in X$, both $x \wedge y=\inf \{x, y\}$ and $x \vee y=\sup \{x, y\}$ exist in $X$. Note that a totally ordered set is a lattice. A nonempty subset $A$ of $X$ is a sublattice if for all $x, y \in A, x \wedge_{X} y, x \vee_{X} y \in A$, where $x \wedge_{X} y$ and $x \vee_{X} y$ are obtained taking the infimum and supremum as elements of $X$ (as opposed to using the relative order on $A$ ).

A lattice $(X, \leq)$ is complete if for every nonempty subset $A$ of $X, \inf A, \sup A$ exist in $X$. A nonempty subset $A$ of $X$ is subcomplete if $B \subseteq A, B \neq \emptyset$ implies $\inf _{X} B, \sup _{X} B \in A$, again taking inf and sup of $B$ as a subset of $X$. For two subsets $A, B$ of $X$, say that $A$ is smaller than $B$ in the strong set order if $a \in A, b \in B$ implies 
$a \wedge b \in A, a \vee b \in B$

Let $(X, \leq)$ be a lattice. Say that a correspondence $\phi: X \rightarrow X$ is weakly increasing over $A \subseteq X$ if $x, y \in A$ and $x \leq y$ implies that there is $z \in \phi(x)$ and $z^{\prime} \in \phi(y)$ with $z \leq z^{\prime}$. Also, say that $\phi$ is increasing in the strong set order if $x<y$ implies that $\phi(x)$ is smaller in the strong set order than $\phi(y)$. Note that when $\phi$ is a function, i.e. single valued, both concepts coincide with the usual notion of "monotone weakly increasing."

A correspondence $\phi: X \rightarrow X$ takes finite values if $\phi(x)$ is a finite set for all $x \in X$.

\subsection{The Model}

All results in the paper are results about functions, or correspondences, on a set $X$. The reader should think of the results as dealing with the best-response function, or correspondence, of a game.

Let $\Gamma=\left\{I,\left\{u_{i}\right\}_{i \in I}\left\{S_{i}\right\}_{i \in I}\right\}$ be a normal-form game. That is, $I$ is a set of players, and each player $i \in I$ is endowed with a strategy space $S_{i}$ and a payoff function $u_{i}$ : $X=\times_{j \in I} S_{j} \rightarrow \mathbf{R}$. If $\beta_{i}: X \rightarrow S_{i}$ is player $i$ 's best-response correspondence, $\beta_{i}(s)=$ $\operatorname{argmax}_{\tilde{s}_{i}} u_{i}\left(\tilde{s}_{i}, s_{-i}\right)$, then $\phi: X \rightarrow X$ defined by $\phi=\times_{i \in I} \beta_{i}$ is the game's best-response correspondence. If best-responses are always unique, then $\beta_{i}$ is a function, and $f=\times_{i \in I} \beta_{i}$ is the game's best-response function. The set of fixed points of $\phi-$ or, if best-responses are unique, of $f$-coincides with the set of pure-strategy Nash equilibria of $\Gamma$.

I shall only deal with pure strategies and pure-strategy equilibria, but one can apply my results to the best-response correspondence of the mixed extension of a game. The interpretation of my results for the mixed extension of a game is straightforward. ${ }^{4}$

A game $\Gamma=\left\{I,\left\{u_{i}\right\}_{i \in I}\left\{S_{i}\right\}_{i \in I}\right\}$ is a game of strategic complementarities (GSC) if there is an order $\leq$ on $X$ such that $(X, \leq)$ is a lattice and $\phi$ is weakly increasing. If $\Gamma$ has unique best-responses, $\Gamma$ is a GSC if there is an order $\leq$ on $X$ such that $(X, \leq)$ is a lattice and $f$ is a monotone increasing function.

It will be useful to interpret some assumptions in terms of Cournot best-response dynamics: If players meet to play $\Gamma$ over and over again, and if they, in each round of

\footnotetext{
${ }^{4}$ I focus on pure strategies because the GSC literature considers usually orders on pure strategies. It may be desirable to order both pure and mixed strategies in a way that is consistent (for example by using the first-order stochastic dominance order on mixed strategies) but unless all strategy spaces are chains, this is incompatible with strategic complementarities, see Echenique (2000).
} 
play $n$, choose a best response to their opponents' play in $n-1$, we say that they follow Cournot best-response dynamics. If $\phi$ is $\Gamma$ 's best-response correspondence, all Cournot best-response dynamics are generated by $x_{n} \in \phi\left(x_{n-1}\right)$ (for some initial $x_{0}$ ).

Definition 1 Let $X$ be a set and $\phi: X \rightarrow X$ be a correspondence. A point $x \in X$ is a cycle of $\phi$ if there is $n \in \mathbf{N}$ such that $x \in \phi^{n}(x)$. Let $X$ be a topological space. A fixed point e is globally stable for $\phi$ if, for every $x \in X$ and every sequence $\left\{x_{k}\right\}$ with $x_{0}=x$ and $x_{k} \in \phi\left(x_{k-1}\right), x_{k} \rightarrow e$. By interpreting $f: X \rightarrow X$ as a correspondence with singleton values, these definitions extended to functions.

Absence of cycles means that no Cournot best-response dynamics will get caught in a cycle. ${ }^{5}$ And global stability means that players that engage in Cournot best-response dynamics will eventually approach Nash equilibrium play.

\section{Unique Equilibrium}

Let $X$ be a set, $f: X \rightarrow X$ a function and $\phi: X \rightarrow X$ a correspondence. First, I consider the problem of when there is an an order $\leq$ such that $(X, \leq)$ is a lattice and $f$ is monotone increasing. Second, I consider the problem of when there is an an order $\leq$ such that $(X, \leq)$ is a lattice and $\phi$ is weakly increasing. Third, I discuss some technical issues.

Theorem 2 Let $f: X \rightarrow X$ have a unique fixed point $e \in X$. There is a total order $\leq$ on $X$ such that $f$ is monotone increasing if and only if $f$ has no cycles besides $e$.

The proof of Theorem 2 follows from Theorem 3 below. Let me say here that: a) It is immediate that the absence of cycles is necessary for the existence of a total order such that $f$ is increasing. For example, if $x=f(f(x))=f^{2}(x)$ and $x \neq f(x)$ then $f$ cannot be monotone increasing, as one and only one of $x<f(x)$ and $f(x)<x$ must hold. Similarly for $x=f^{n}(x), n \geq 2$. b) When $X$ is finite it is easy to prove sufficiency, the idea is as follows. Absence of cycles implies that for each $x$ there is an $n$ such that $e=f^{n}(x)$; now let $x<y$ whenever the smallest $n$ such that $e=f^{n}(x)$ is larger than the smallest $n$ such that $e=f^{n}(y)$, this ensures that $f(x)<f(y)$. It remains to order the elements that are

\footnotetext{
${ }^{5}$ Cournot best-response dynamics is a very naive learning model, my results do not depend of any virtues of Cournot dynamics as a learning model. The absence of cycles should be viewed as a technical condition. It is true, though, that Cournot dynamics is easy to implement on a computer, and therefore the condition of absence of cycles is easy to verify computationally.
} 


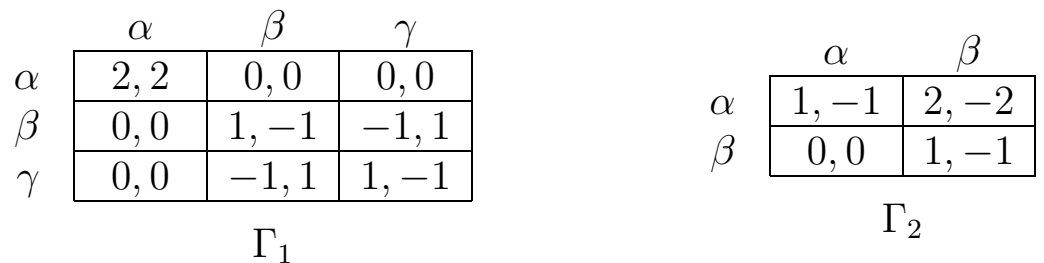

Figure 2: A non-GSC, and a GSC that is zero-sum.

the same number of iterates away from $e$, which is slightly cumbersome but easy (see the proof of Theorem 3 for the details, and for the proof when $X$ is infinite).

Theorem 2 may have some mathematical interest, independently of the application to game theory that I emphasize.

Absence of cycles is a non-topological condition that is weaker than global stability: global stability implies the absence of cycles other than $e$, but absence of cycles does not imply global stability - for example, let $X$ be the unit disk in $\mathbf{R}^{2}$ and $f$ be a rotation of $X$ by an irrational number, then $f$ has a unique fixed point, $(0,0)$, and no cycles, but $(0,0)$ is clearly not globally stable. The existence of an order such that $f$ is increasing is a non-topological statement, so it cannot depend on global stability.

\subsection{Examples}

Let $\Gamma=\left\{I,\left\{u_{i}\right\}_{i \in I}\left\{S_{i}\right\}_{i \in I}\right\}$ be a normal-form game with a unique Nash equilibrium. If all players have unique best-responses - which, as these are pure-strategy best-responses, is generically the case when $\Gamma$ is a finite game - then, by Theorem 2, $\Gamma$ is a GSC if and only if Cournot best-response dynamics have no cycles except for $e .{ }^{6}$ In particular, if $e$ is globally stable under Cournot best-response dynamics, then $\Gamma$ is a GSC. One implication of these results is that a game that satisfies the dominant diagonal condition in Gabay and Moulin (1980), and has thus a unique, globally stable, equilibrium, is a GSC.

As an illustration of Theorem 2, consider $\Gamma_{1}$, the $3 X 3$ game on the left in Figure 2 . This game has a unique pure-strategy equilibrium, and best-response dynamics have the cycles $(\alpha, \alpha) \rightarrow(\alpha, \alpha),(\beta, \alpha) \rightarrow(\alpha, \gamma) \rightarrow(\gamma, \alpha) \rightarrow(\alpha, \beta) \rightarrow(\beta, \alpha)$, and $(\beta, \beta) \rightarrow$ $(\beta, \gamma) \rightarrow(\gamma, \gamma) \rightarrow(\gamma, \beta) \rightarrow(\beta, \beta)$. Therefore there is no order on strategies such that the game in Figure 2 is a GSC.

\footnotetext{
${ }^{6}$ See section 3.3 for the difference between lattice and totally ordered strategy spaces.
} 
Consider $\Gamma_{2}$, the zero-sum game on the right in Figure 2 as a second illustration of Theorem 2. This is a GSC because it has a unique equilibrium and no cycles. The intuitive idea that zero-sum games and GSC are different is therefore false.

\section{$3.2 \quad$ Non-unique best-responses}

Theorem 2 for functions can be generalized to correspondences with finite values, but the characterization is not completely tight. Absence of cycles implies the existence of a total order that makes the correspondence weakly monotone, but a weakly monotone correspondence may have cycles, as weak monotonicity does not control all selections from the correspondence.

Theorem 3 Let $\phi: X \rightarrow X$ have a unique fixed point $e \in X$. If $\phi$ takes finite values and has no cycles besides e, there is a total order on $X$ such that $\phi$ is weakly increasing. Further, if $\{e\}=\phi(e)$ and there is a total order on $X$ such that $\phi$ is increasing in the strong set order, then $\phi$ has no cycles besides e.

The proof of Theorem 3 is in section 9 .

I need the hypothesis of a correspondence with finite values to use non-standard analysis the way I do. If $\Gamma$ is a game with unique best-responses, of course the bestresponses have finite values, and both weakly increasing and increasing in the strong set order coincide with monotone increasing. So Theorem 3 implies Theorem 2. Also, if $\Gamma$ is a finite game, then best-responses take finite values. I have not been able to extend the characterization in Theorem 3 to arbitrary games with infinite strategy spaces and non-unique best-responses.

Global stability implies absence of cycles, thus we obtain

Corollary 4 Let $X$ be a topological space and $\phi: X \rightarrow X$ a correspondence with finite values and a unique fixed point $e \in X$. If $e$ is globally stable then there is a total order on $X$ such that $\phi$ is weakly increasing.

\subsection{Totally ordered strategy space vs. a lattice strategy space, and completeness.}

Theorems 2 and 3 give necessary and sufficient conditions for a game to be a GSC with a totally ordered strategy space. I defined GSC as games with lattice strategy spaces, 


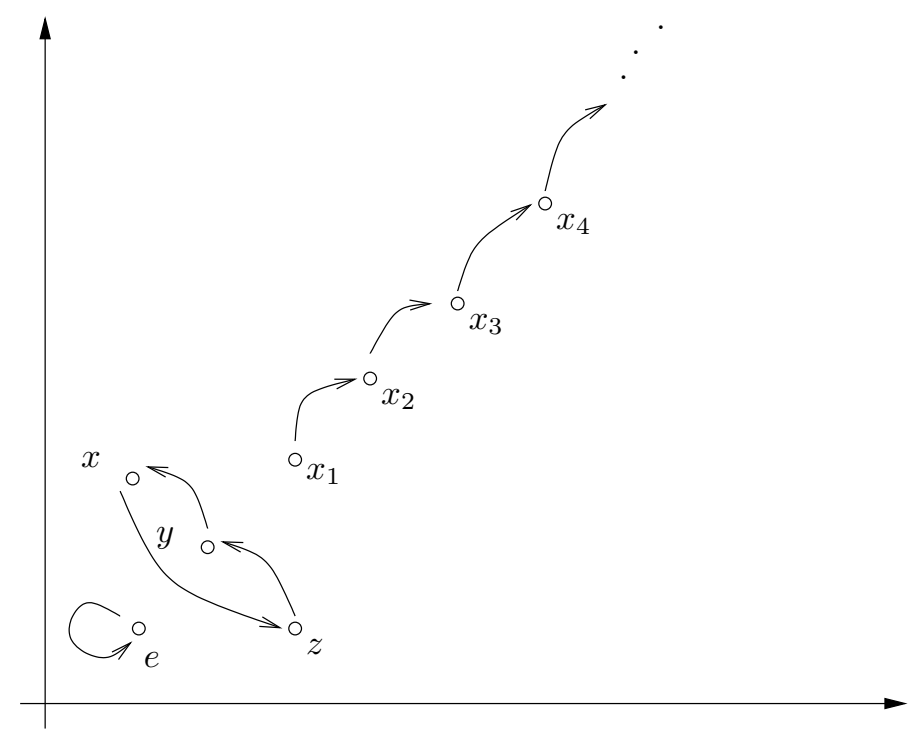

Figure 3: Arrows show action of $f:\left\{e, x, y, z, x_{1}, x_{2}, \ldots\right\} \rightarrow\left\{e, x, y, z, x_{1}, x_{2}, \ldots\right\}$

is there some loss in focusing on totally ordered strategies? Yes, but it is essentially a technical problem: Consider Theorem 2. If a game's best-response function does not have cycles, then the game is a GSC because there is a total order on strategies such that best responses are monotone increasing, and a totally ordered set is a lattice. On the other hand, if $\Gamma$ is a GSC where each $S_{i}$ is a complete lattice, and best-responses $f$ are continuous, then a unique Nash equilibrium is globally stable (Vives 1990, Milgrom and Roberts 1990) so $f$ has no cycles and there is a total order on strategies such that $f$ is increasing. In particular this implies that, in finite games, a game with a unique equilibrium is a GSC if and only if best responses have no cycles.

In GSC where strategy spaces are non-complete lattices, best-responses may have cycles. Consider the example in Figure 3. The arrows in the figure show the action of a function $f:\left\{e, x, y, z, x_{1}, x_{2}, \ldots\right\} \rightarrow\left\{e, x, y, z, x_{1}, x_{2}, \ldots\right\}$. Let the infinite set $\left\{e, x, y, z, x_{1}, x_{2}, \ldots\right\}$ be ordered as a subset of $\mathbf{R}^{2}$. The function $f$ has a unique fixed point, $e$, a cycle $z \rightarrow y \rightarrow x \rightarrow z$, and it is monotone increasing. The example works because Cournot best-response dynamics starting at $x_{1}$ (or any other point larger than $x, y$ and $z$ ) is a monotone increasing sequence $x_{1}, x_{2}, \ldots$ that does not converge to a fixed point different from $e$. So the characterization in Theorem 2 is not completely tight. As I remarked earlier, complementarities is a non-topological condition, so the characterization must be independent of completeness of strategy spaces. In a sense, then, it is impossible to avoid problems like the one in Figure 3. 
In the usual definition of GSC - supermodular games - there is a link between the order and the topology on the strategy spaces that, among other things, ensures the existence of equilibria (by Tarski's Theorem), and that if equilibrium is unique it must be globally stable. This link is (order) completeness. Here I construct orders that make best-responses monotone increasing, but that do not have any relation to the topology on the strategy spaces. In finite games this does not matter: a finite game without equilibria cannot be a GSC, and a game with a unique equilibrium is a GSC if and only if this equilibrium is globally stable. In infinite games, though, there may be GSC without equilibria, and GSC with a unique equilibrium that is not globally stable. I wish to emphasize that, despite this technical problem, for games with multiple equilibria, all of Vives's, Topkis's and Milgrom and Roberts's results hold trivially with the order that I construct in section 4 .

\section{Multiple Equilibria}

A game with two or more Nash equilibria is (almost trivially) a GSC: set one equilibrium $\underline{e}$ as the smallest strategy profile, another equilibrium $\bar{e}$ as the largest, and let all other strategy profiles $x$ be unordered, except for satisfying $\underline{e} \leq x \leq \bar{e}$. I work out the details in Theorem 5, but besides answering the question in this paper, such a coarse order is uninteresting.

For finite games I can do much better; I can find an order such that the game is a GSC, and such that the interval prediction is as sharp as is possible. Theorem 7 below says that, if a function $f$ on a finite set has at least two different fixed points, then there is a lattice order such that $f$ is monotone increasing, the interval prediction is the order interval between the fixed points, and any other lattice order that does the same work must have a larger interval prediction.

Theorem 5 Let $\phi: X \rightarrow X$ have at least two different fixed points. There is an order $\leq$ on $X$ such that $(X, \leq)$ is a complete lattice, $\phi$ is weakly increasing, and the set of fixed points of $\phi$ is a complete sublattice of $X$.

Proof. Let $\underline{e}, \bar{e}$ be different fixed points of $\phi$. Define $\leq$ on $X$ by $x \leq y$ if and only if one of the following is true: $x=y, x=\underline{e}$, or $y=\bar{e}$. Then, for any non-singleton $A \subseteq X$, $\underline{e}$ is the unique lower bound on $A$, hence $\inf A=\underline{e}$; similarly sup $A=\bar{e}$ as $\bar{e}$ is the unique 
upper bound on $A$. Thus, for all $x, y \in X, x \vee y, x \wedge y \in X$, and for all non-empty $A \subseteq X$, $\inf A, \sup A \in X$, so $X$ is a complete lattice.

Now, let $x \leq y$ and $x \neq y$; if $x=\underline{e}$ then $\underline{e} \in \phi(x)$, so $\underline{e} \leq z$ for all $z \in \phi(y)$, and similarly if $y=\bar{e}$. If $x=y$ then any $z=z^{\prime} \in \phi(x)=\phi(y)$ satisfies $z \leq z^{\prime}$. Thus if $x \leq y$ there is $z \in \phi(x)$ and $z^{\prime} \in \phi(y)$ with $z \leq z^{\prime}$, so $\phi$ is weakly increasing.

Finally, if $A$ is a non-singleton set of fixed points, then $\underline{e}$ is the unique lower bound, and $\bar{e}$ is the unique upper bound, on $A$. So, $\inf A=\underline{e}$ and $\sup A=\bar{e}$. But $\underline{e}$ and $\bar{e}$ are fixed points, so the set of fixed points is a complete sublattice of $X$.

\section{Remark.}

1. The usual definitions of GSC ensure that best-responses are increasing in the strong set order, and that $\phi$ takes subcomplete- sublattice-values. The order that I construct in Theorem 5 does not guarantee that $\phi$ has these properties (unless, of course, $\phi$ is a function), but Topkis's, Vives's, Milgrom and Roberts's, and Milgrom and Shannon's results are (trivially) true with the constructed order.

2. By Zhou's version of Tarski's Fixed Point Theorem the equilibrium set of a GSC is a complete lattice. Topkis, Vives and Zhou present examples where it is not a sublattice (see Echenique (2001), though). By Theorem 5 there is a partial order on $X$ such that this equilibrium set is a complete sublattice, without eliminating complementarities.

For clarity, I include the statement of Theorem 5 when $\phi$ is a function as:

Corollary 6 Let $f: X \rightarrow X$ have at least two different fixed points. There is an order $\leq$ on $X$ such that $(X, \leq)$ is a complete lattice, $f$ is monotone increasing, and the set of fixed points of $f$ is a complete sublattice of $X$.

Theorem 7 Let $f: X \rightarrow X$ be a function on a finite set $X$ with at least two different fixed points, $\underline{e}$ and $\bar{e}$. There is a partial order $\leq$ on $X$ such that

1. $(X, \leq)$ is a complete lattice and $f$ is monotone increasing;

2. $\underline{e}$ is the smallest, and $\bar{e}$ the largest, fixed point of $f$;

3. if $\preceq$ is any other partial order on $X$ that satisfies statements 1 and 2 of this theorem, then, for any $z \in X, \underline{e} \leq z \leq \bar{e}$ implies that $\underline{e} \preceq z \preceq \bar{e}$. 
Remark. Item 3 in the Theorem says that the order $\leq$ is - from the viewpoint of the interval prediction - the best order possible: any other order that makes $f$ a GSC must involve a larger (less precise) interval prediction.

I leave the proof of Theorem 7 for Section 9. Let me say here that the proof is constructive, so it works by finding a $\leq$ that satisfies the statements in the Theorem. The order $\leq$ leaves out of the interval prediction (among other things) all elements $x$ in the basin of either $\underline{e}$ or $\bar{e}$, where $x$ is in the basin of a fixed point $e$ if there is some $n \in \mathbf{N}$ such that $e=f^{n}(x)$.

\section{Discussion of the interval prediction.}

I show that there are games for which the interval prediction is unavoidably vacuous, and that there are games for which the interval prediction must contain strictly dominated strategies.

The discussion of these games complements the message of Theorem 7 . Theorem 7 delivers an order with the best possible interval prediction, but the construction of this order is in general quite cumbersome. I believe the examples that follow will help understand what can and cannot be left out of the interval prediction.

1. Consider the coordination game in Figure 1. I shall show that there is no order on strategies that preserves complementarities, and where the interval prediction is sharper than the whole strategy space. Let

$$
b=b_{1} \times b_{2}:\{\alpha, \beta\}^{2} \rightarrow\{\alpha, \beta\}^{2}
$$

be the game's best-response function, and $\leq$ be an order on $\{\alpha, \beta\}^{2}$ such that $b$ is monotone increasing. Now, it must be that $(\alpha, \beta)$ and $(\beta, \alpha)$ are incomparable under $\leq$. To see this, let $(\alpha, \beta)<(\beta, \alpha)$. Then $b(\beta, \alpha)=(\alpha, \beta)<b(\alpha, \beta)=(\beta, \alpha)$, so $b$ would not be increasing. Similarly if $(\beta, \alpha)<(\alpha, \beta)$. Then, for $\left(\{\alpha, \beta\}^{2}, \leq\right)$ to be a lattice, it must be that $(\alpha, \beta) \vee(\beta, \alpha)$ and $(\alpha, \beta) \wedge(\beta, \alpha)$ equals either $(\alpha, \alpha)$ and $(\beta, \beta)$ or, respectively, $(\beta, \beta)$ and $(\alpha, \alpha)$. But $(\alpha, \beta) \vee(\beta, \alpha) \neq(\alpha, \beta) \wedge(\beta, \alpha)$, hence either $(\alpha, \alpha)$ is the smallest strategy profile and $(\beta, \beta)$ is the largest, or vice versa.

2. I show that this does not depend on the coordination game being $2 X 2$, it depends on the cycles in best-responses between the non-equilibrium strategy profiles $(\alpha, \beta)$ and 


\begin{tabular}{l|c|c|c|c|}
\multicolumn{1}{c}{} & \multicolumn{1}{c}{$\alpha$} & \multicolumn{1}{c}{$\beta$} & $\gamma$ & \multicolumn{1}{c}{$\delta$} \\
\cline { 2 - 5 }$\alpha$ & 2,2 & 0,0 & 0,0 & 0,0 \\
\cline { 2 - 5 }$\beta$ & 0,0 & 0,1 & 1,0 & 0,0 \\
\cline { 2 - 5 }$\gamma$ & 0,0 & 1,0 & 0,1 & 0,0 \\
\cline { 2 - 5 }$\delta$ & 0,0 & 0,0 & 0,0 & 1,1 \\
\cline { 2 - 5 } & & &
\end{tabular}

Figure 4: $\Gamma_{3}$, a $4 \mathrm{X} 4$ coordination game.

$(\beta, \alpha) .{ }^{7}$ In fact, consider the $4 X 4$ coordination game $\Gamma_{3}$ in Figure 4. In this game, best-responses have three cyclical orbits (besides the fixed-point cycles $(\alpha, \alpha) \rightarrow(\alpha, \alpha)$ and $(\delta, \delta) \rightarrow(\delta, \delta))$ :

$$
\begin{aligned}
& (\beta, \alpha) \rightarrow(\alpha, \beta) \rightarrow(\gamma, \alpha) \rightarrow(\alpha, \gamma) \rightarrow(\beta, \alpha), \\
& (\delta, \gamma) \rightarrow(\beta, \delta) \rightarrow(\delta, \beta) \rightarrow(\gamma, \delta) \rightarrow(\delta, \gamma), \\
& \text { and }(\beta, \beta) \rightarrow(\gamma, \beta) \rightarrow(\gamma, \gamma) \rightarrow(\beta, \gamma) \rightarrow(\beta, \beta) .
\end{aligned}
$$

Then, if a partial order $\leq$ makes the $4 X 4$ coordination game into a GSC, no strategy that belongs to a cyclical orbit can be the smallest, or the largest, strategy profile of the game. To see this, suppose, for example, that $(\beta, \alpha)$ is the smallest strategy profile. Then $(\beta, \alpha)<(\alpha, \beta)$, so if $b$ is monotone increasing, $b^{3}(\beta, \alpha) \leq b^{3}(\alpha, \beta)$. But $(\beta, \alpha)=b^{3}(\alpha, \beta)$ and $(\alpha, \beta)=b^{3}(\beta, \alpha)$. So it must be that the smallest and largest element in the strategy space is $(\alpha, \alpha)$ and $(\delta, \delta)$, as a finite lattice has a smallest and a largest element.

3. All strategies in the coordination games are rationalizable. One could then conjecture that the interval prediction must coincide with the rationalizable strategies, and that the problem with the coordination games is that all strategies are rationalizable. The conjecture would be false, I present an example of a game where one of the player's dominated strategies must be in the interval prediction.

Consider $\Gamma_{4}$, the example in Figure 5. $\Gamma_{4}$ is a three-player game. The strategy space of each player $i$ is $\{\alpha, \beta\}$; the game is represented in two matrices, player 1 chooses rows, player 2 chooses columns, and player 3 chooses matrices. In each entry of a matrix, the first number is the payoff to player 1, the second number is the payoff to player 2, and the third number is the payoff to player 3.

Let $b$ be $\Gamma_{4}$ 's best-response function, and $X=\{\alpha, \beta\}^{3}$ be the strategy space. Note that $b$ has exactly two fixed points, $(\alpha, \alpha, \beta)$ and $(\beta, \beta, \beta)$. Note also that $\alpha$ is a strictly

\footnotetext{
${ }^{7}$ In both coordination games, all strategy profiles are rationalizable. So, it follows from Milgrom and Roberts's results that all strategy profiles are in the interval prediction. I show this directly here and in item 1 to make explicit the role of cycles.
} 


\begin{tabular}{|c|c|c|c|c|c|}
\hline \multirow{3}{*}{$\begin{array}{l}\alpha \\
\beta\end{array}$} & $\alpha$ & $\beta$ & \multirow{3}{*}{$\begin{array}{l}\alpha \\
\beta\end{array}$} & \multicolumn{2}{|l|}{$\alpha$} \\
\hline & $1,0,0$ & $1,1,0$ & & $1,1,1$ & $0,0,1$ \\
\hline & $0,0,0$ & $0,1,0$ & & $0,0,1$ & $1,1,1$ \\
\hline
\end{tabular}

Figure 5: $\Gamma_{4}$, a three-player game.

dominated strategy for player 3 . There is a cycle $(\alpha, \beta, \beta) \rightarrow(\beta, \alpha, \beta) \rightarrow(\alpha, \beta, \beta)$. And $b\left(s_{1}, s_{2}, \alpha\right)=(\alpha, \beta, \beta)$ for all $s_{1}, s_{2} \in\{\alpha, \beta\}$.

Let $\leq$ be an order that makes $\Gamma_{4}$ into a GSC. Repeating the arguments used for the coordination games, $(\alpha, \beta, \beta)$ and $(\beta, \alpha, \beta)$ must be unordered because they are cycles. So, $(\alpha, \beta, \beta)$ and $(\beta, \alpha, \beta)$ cannot be either $\inf _{\leq} X$ or $\sup _{\leq} X$. I claim that, for all $s_{1}, s_{2} \in$ $\{\alpha, \beta\},\left(s_{1}, s_{2}, \alpha\right) \neq \inf _{\leq} X$ and $\left(s_{1}, s_{2}, \alpha\right) \neq \sup _{\leq} X$. Suppose that some $\left(s_{1}, s_{2}, \alpha\right)=$ $\inf _{\leq} X$. Then $\left(s_{1}, s_{2}, \alpha\right) \leq(\alpha, \beta, \beta)$. But $b$ is monotone increasing, thus

$$
(\alpha, \beta, \beta)=b\left(s_{1}, s_{2}, \alpha\right) \leq b(\alpha, \beta, \beta)=(\beta, \alpha, \beta),
$$

impossible since $(\alpha, \beta, \beta)$ and $(\beta, \alpha, \beta)$ must be unordered. Similarly, $\left(s_{1}, s_{2}, \alpha\right) \neq \sup _{\leq} X$, for all $s_{1}, s_{2} \in\{\alpha, \beta\}$. Then $(\alpha, \alpha, \beta)=\inf _{\leq} X$ and $(\beta, \beta, \beta)=\sup _{\leq} X$ (or vice-versa).

Thus, any order that makes $\Gamma_{4}$ into a GSC must give all $X$ as the interval prediction. Not only is the interval prediction vacuous, it does not rule out player 3 choosing $\alpha$, a dominated strategy.

\section{Comparative Statics}

In a parameterized GSC, if a parameter $t$ is complementary to players' choices, there are selections of equilibria that are monotone increasing in $t$ (Lippman, Mamer, and McCardle 1987, Sobel 1988, Villas-Boas 1997, Milgrom and Roberts 1990, Milgrom and Roberts 1994, Milgrom and Shannon 1994, Echenique 2002, Echenique and Sabarwal 2000). By theorems 3 and 5, most games can be rationalized as GSC. A natural question is: can any comparative statics conclusion be rationalized as coming from a parameterized GSC? The answer is no, comparative statics conclusions act as an "identifying condition" that restricts the choice of an order on strategy spaces.

Using the framework in Milgrom and Roberts (1994), the question can be phrased as follows. Let $f_{t}, f_{t^{\prime}}: X \rightarrow X$, let $e$ be a fixed point of $f_{t}$, and $e^{\prime}$ a fixed point of $f_{t^{\prime}}$. Is there an order on $X$ such that $e<e^{\prime}$ and such that $f_{t}(x) \leq f_{t^{\prime}}(x)$ for all $x$ ? 


\begin{tabular}{l|c|c|} 
& \multicolumn{1}{c}{$\alpha$} & \multicolumn{1}{c}{$\beta$} \\
\cline { 2 - 3 }$\alpha$ & $a, b$ & $c, d$ \\
\cline { 2 - 3 }$\beta$ & $e, f$ & $g, h$ \\
\cline { 2 - 3 } & &
\end{tabular}

$\Gamma_{4}$

\begin{tabular}{l|c|c|}
\multicolumn{1}{c}{} & \multicolumn{1}{c}{$\alpha$} & \multicolumn{1}{c}{$\beta$} \\
\cline { 2 - 3 }$\alpha$ & 1,1 & 0,1 \\
\cline { 2 - 3 }$\beta$ & 0,1 & 1,0 \\
\hline
\end{tabular}

$\Gamma_{5}$

Figure 6: $\Gamma_{3}$ used in the proof of, and $\Gamma_{4}$ illustrates, Proposition 8

In general the answer is no. Consider the following example, let $x, e, e^{\prime} \in X$ with $e=f_{t}(e), e^{\prime}=f_{t^{\prime}}\left(e^{\prime}\right)$ and $e \neq e^{\prime}$. Suppose that $e^{\prime}=f_{t}(x)$ and $e=f_{t^{\prime}}(x)$. If there is an order $\leq$ such that $f_{t}(z) \leq f_{t^{\prime}}(z)$ for all $z \in X$, then $e=f_{t}(e) \leq f_{t^{\prime}}(e)=e^{\prime}$ and $e<e^{\prime}$ because $e \neq e^{\prime}$. But $e^{\prime}=f_{t}(x) \leq f_{t^{\prime}}(x)=e$, a contradiction.

The example above shows that we cannot rationalize comparative statics conclusions by a model where the parameter is complementary to players' choices. Alternatively, is there an order on $X$ such that $e<e^{\prime}$ and such that $f_{t}$ and $f_{t^{\prime}}$ are monotone increasing functions? That is, can we rationalize a comparative statics conclusion as coming from parameterized GSC, even though the parameter may not be complementary to the strategies? The following example shows that this need not be the case. Let $x, e, e^{\prime} \in X$ with $e=f_{t}(e), e^{\prime}=f_{t^{\prime}}\left(e^{\prime}\right)$ and $e \neq e^{\prime}$. Suppose that $x=f_{t}\left(e^{\prime}\right), e=f_{t^{\prime}}(x)$ and $e^{\prime}=f_{t^{\prime}}(e)$. Then $e<e^{\prime}$ implies that $f_{t}(e) \leq f_{t}\left(e^{\prime}\right)$, so $e<x$. Then $f_{t^{\prime}}(e) \leq f_{t^{\prime}}(x)$ implies that $e^{\prime}<e$, a contradiction.

\section{Application 1: $2 X 2$ Games}

As an application of Theorems 3 and 5 I show that, generically, $2 X 2$ games either have no equilibria or have complementarities. Essentially, then, $2 X 2$ games are either isomorphic to "Matching Pennies", or they are GSC.

Proposition 8 Generically, a $2 X 2$ game either has no pure-strategy Nash equilibrium, or it is a GSC.

Proof. Consider the game in Figure 6 on the left. Suppose that $a, b, c$, etc. are such that $(\beta, \beta)$ is the unique Nash equilibrium, and $(\beta, \beta)$ is a strict equilibrium, so $c<g$ and $f<h$. I will rule out all possible cycles in best-response dynamics, except for the cycle involving $(\beta, \beta)$. Then Theorem 2 implies that the game is a GSC.

Since $c<g$ and $f<h,(\alpha, \alpha)$ is not a best response to $(\alpha, \beta)$ or to $(\beta, \alpha)$. This rules out the cycles $(\alpha, \alpha) \rightarrow(\alpha, \beta) \rightarrow(\alpha, \alpha),(\alpha, \beta) \rightarrow(\alpha, \alpha) \rightarrow(\alpha, \beta),(\alpha, \alpha) \rightarrow$ 
$(\beta, \alpha) \rightarrow(\alpha, \alpha),(\beta, \alpha) \rightarrow(\alpha, \alpha) \rightarrow(\beta, \alpha),(\alpha, \alpha) \rightarrow(\beta, \alpha) \rightarrow(\alpha, \beta) \rightarrow(\alpha, \alpha)$, and $(\alpha, \alpha) \rightarrow(\alpha, \beta) \rightarrow(\beta, \alpha) \rightarrow(\alpha, \alpha)$.

Finally, the cycle $(\beta, \alpha) \rightarrow(\alpha, \beta) \rightarrow(\beta, \alpha)$ requires that $a \geq e$ and $b \geq d$. But then $(\alpha, \alpha)$ would be an equilibrium, contradicting that $(\beta, \beta)$ is the unique Nash equilibrium. Similarly for $(\alpha, \beta) \rightarrow(\beta, \alpha) \rightarrow(\alpha, \beta)$. These are all possible cycles.

We have shown that all $2 X 2$ games with a unique, strict Nash equilibrium are GSC. All games with more than one equilibrium are GSC by Theorem 5 . The property that a unique equilibrium is strict is generic in the class of $2 X 2$ games.

Proposition 8 does not extend to more complex games than $2 X 2$, see the example in Figure 2 on the left. The "genericity" qualification in Proposition 8 is necessary. Consider the game on the right in Figure 6. This game has a unique Nash equilibrium $(\alpha, \alpha)$ in pure strategies, and a cycle, $(\alpha, \alpha) \rightarrow(\alpha, \beta) \rightarrow(\beta, \alpha) \rightarrow(\alpha, \alpha)$. The cycle is produced by player 2's indifference when 1 chooses $\alpha$, which is non-generic.

\section{Application 2: Ordinal Potential Games}

A game $\Gamma=\left\{I,\left\{u_{i}\right\}_{i \in I}\left\{S_{i}\right\}_{i \in I}\right\}$ is an ordinal potential game if there is a function $P: S=\times_{i \in I} S_{i} \rightarrow \mathbf{R}$ such that

$$
u_{i}\left(s_{i}, s_{-i}\right)<u_{i}\left(s_{i}^{\prime}, s_{-i}\right) \text { if and only if } P\left(s_{i}, s_{-i}\right)<P\left(s_{i}^{\prime}, s_{-i}\right),
$$

for all $s_{i}, s_{i}^{\prime} \in S_{i}, s_{-i} \in S_{-i}$, and $i \in I$. Potential games were studied in detail by Monderer and Shapley (1996) (see their paper for references to earlier work on potential games).

My results shed some light on the relation between GSC and ordinal potential games. I show that, generically, a finite two-player ordinal potential game is a GSC; and that ordinal potential games with more than two players need not be GSC. ${ }^{8}$

Proposition 9 Let $\Gamma$ be a finite two-player game with unique best responses. If $\Gamma$ is an ordinal potential game, then it is a GSC.

Proposition 9 does not extend to games with more than two players. For a counterexample, consider the three-player game in Figure 7. Here, player 1 chooses a strategy in

\footnotetext{
${ }^{8}$ Further, it is easy to see that GSC need not be ordinal potential games. I do not discuss this here, the counterexamples are very simple.
} 


\begin{tabular}{c|c|c|}
\multicolumn{1}{c}{} & \multicolumn{1}{c}{$\alpha$} & $\beta$ \\
\cline { 2 - 3 }$\alpha$ & $-1,-1,-1$ & $-1,-1,-1$ \\
\cline { 2 - 3 }$\beta$ & $1,1,0$ & $0,0,1$ \\
\cline { 2 - 3 }$\gamma$ & $0,0,1$ & $1,1,0$ \\
\hline
\end{tabular}

$\alpha$

\begin{tabular}{l|c|c|}
\multicolumn{1}{c}{} & \multicolumn{1}{c}{$\alpha$} & $\beta$ \\
\cline { 2 - 3 }$\alpha$ & $1,1,1$ & $1,0,1$ \\
\cline { 2 - 3 }$\beta$ & $0,1,1$ & $0,0,0$ \\
\cline { 2 - 3 }$\gamma$ & $0,0,0$ & $0,1,1$ \\
\hline & \multicolumn{2}{|c}{$\beta$}
\end{tabular}

Figure 7: A three-player ordinal potential game that is not a GSC.

$S_{1}=\{\alpha, \beta, \gamma\}$ (rows), player 2 chooses a strategy in $S_{2}=\{\alpha, \beta\}$ (columns), and player 3 a strategy in $S_{3}=\{\alpha, \beta\}$ (matrices). The payoffs are indicated in the figure.

The game in Figure 7 has a unique equilibrium, $(\alpha, \alpha, \beta)$, and the best-response cycle $(\beta, \beta, \alpha) \rightarrow(\gamma, \alpha, \alpha) \rightarrow(\beta, \beta, \alpha)$ By Theorem 2, then, there is no order on strategies so that his game is a GSC. But by Monderer and Shapley's results, the game has a generalized ordinal potential, as it has the finite improvement property (this is a bit cumbersome to check). Now just perturb the game a bit, it still will not be a GSC because the best-response cycle will persist, and it will be an ordinal potential game by Monderer and Shapley's Corollary 2.6.

Proof of Proposition 9. To prove Proposition 9, I need two definitions and two lemmas. Let $\Gamma=\left\{S_{1}, S_{2}, u_{1}, u_{2}\right\}$ be a two-player game with unique best-responses, and let $\beta\left(s_{1}, s_{2}\right)=\left(\beta_{1}\left(s_{2}\right), \beta_{2}\left(s_{1}\right)\right)$ be $\Gamma$ 's best-response function-note the minor change in notation. A best-response cycle of $\Gamma$ is a sequence $\left(s^{0}, s^{1}, \ldots s^{n}\right)$ in $S=S_{1} \times S_{2}$, where $s^{k}=\beta\left(s^{k-1}\right), 1 \leq k \leq n, s^{0}=s^{n}$, and $s^{k-1} \neq s^{k}, 1 \leq k \leq n$. The use of the term "cycle" here is inconsistent with the rest of the paper, but no confusion should arise because it will be clear, at all times in this section, that a cycle is the whole path $\left(s^{0}, s^{1}, \ldots s^{n}\right)$, not a point in the range of the path.

An infinite improvement path in $S$ is a sequence $\left(w^{0}, w^{1}, \ldots\right)$ such that:

- $\left(w^{0}, w^{1}, \ldots\right)$ is not eventually constant (i.e. there is no $K$ such that the sequence $\left(w^{K}, w^{K+1}, \ldots\right)$ is constant $)$.

- $w^{k-1}$ and $w^{k}$ differ in at most one component,

- if $w^{k-1}$ and $w^{k}$ differ, and $i$ is the player that changes strategy between $w^{k-1}$ and $w^{k}$, then $u_{i}\left(w^{k-1}\right)<u_{i}\left(w^{k}\right)$.

The definition of an infinite improvement path differs from the one in Monderer and Shapley. However, it is immediate to modify their Lemma 2.3 to show: 
Lemma 10 (Monderer and Shapley (1996)) An ordinal potential game cannot have an infinite improvement path.

I do not include a proof of Lemma 10 in this paper.

Lemma 11 Let $\Gamma$ be a two-player game with unique best responses and a unique Nash equilibrium. If $\Gamma$ has a best-response cycle, then it has an infinite improvement path.

Proof. Let $\left(s^{0}, s^{1}, \ldots s^{n}\right)$ be a best-response cycle. We must have $n \geq 2$, or $\left(s^{0}, s^{1}, \ldots s^{n}\right)$ is a constant sequence.

I claim that either $s_{1}^{0} \neq s_{1}^{2}, s_{2}^{0} \neq s_{2}^{2}$, or both hold. Suppose, by way of contradiction, that $s_{1}^{0}=s_{1}^{2}$ and $s_{2}^{0}=s_{2}^{2}$. Then $s_{1}^{0}=\beta_{1}\left(s_{2}^{1}\right)$ and $s_{2}^{0}=\beta_{2}\left(s_{1}^{1}\right)$. Now $s_{1}^{1}=\beta_{1}\left(s_{2}^{0}\right)$ and $s_{2}^{1}=\beta_{2}\left(s_{1}^{0}\right)$ imply that $\left(s_{1}^{0}, s_{2}^{1}\right)$ and $\left(s_{1}^{1}, s_{2}^{0}\right)$ are Nash equilibria. But there is a unique Nash equilibrium, so $\left(s_{1}^{0}, s_{2}^{1}\right)=\left(s_{1}^{1}, s_{2}^{0}\right)$; impossible, as $\left(s_{1}^{0}, s_{2}^{0}\right)=s^{0} \neq s^{1}=\left(s_{1}^{1}, s_{2}^{1}\right)$. Suppose, without loss of generality, that $s_{2}^{0} \neq s_{2}^{2}$.

Extend the sequence $\left(s^{0}, s^{1}, \ldots s^{n}\right)$ to the infinite sequence

$$
\left(s^{0}, s^{1}, \ldots s^{n-1}, s^{0}, s^{1}, \ldots\right) .
$$

So, $s_{1}^{n+1}$ refers to $s_{1}^{1}, s_{2}^{2 n+3}$ to $s_{2}^{3}$, and so on. Construct the sequence

$$
w=\left(w^{0}, w^{1}, w^{2}, \ldots\right)=\left(\left(s_{1}^{1}, s_{2}^{0}\right),\left(s_{1}^{1}, s_{2}^{2}\right),\left(s_{1}^{3}, s_{2}^{2}\right),\left(s_{1}^{3}, s_{2}^{4}\right),\left(s_{1}^{5}, s_{2}^{4}\right),\left(s_{1}^{5}, s_{2}^{6}\right), \ldots\right) .
$$

I shall show that $w$ is an infinite improvement path.

If $n$ is odd, we can construct the following finite sequences:

$$
\begin{aligned}
y^{0}=\left(s_{1}^{1}, s_{2}^{0}\right) & z^{0}=\left(s_{1}^{0}, s_{2}^{1}\right) \\
y^{1}=\left(s_{1}^{1}, s_{2}^{2}\right) & z^{1}=\left(s_{1}^{2}, s_{2}^{1}\right) \\
y^{2}=\left(s_{1}^{3}, s_{2}^{2}\right) & z^{2}=\left(s_{1}^{2}, s_{2}^{3}\right) \\
y^{3}=\left(s_{1}^{3}, s_{2}^{4}\right) & z^{3}=\left(s_{1}^{4}, s_{2}^{3}\right) \\
\vdots & \vdots \\
y^{n-1}=\left(s_{1}^{n}, s_{2}^{n-1}\right)=\left(s_{1}^{0}, s_{2}^{n-1}\right) & z^{n-1}=\left(s_{1}^{n-1}, s_{2}^{n}\right)=\left(s_{1}^{n-1}, s_{2}^{0}\right)
\end{aligned}
$$

If $n$ is even, we shall only need the $y$ sequence as constructed, with the modification that we get $y^{n-1}=\left(s_{1}^{n-1}, s_{2}^{n}\right)=\left(s_{1}^{n-1}, s_{2}^{0}\right)$.

It is easy, if somewhat cumbersome, to show that: If $n$ is odd, then

$$
w=\left(y^{0}, y^{1}, \ldots y^{n-1}, z^{0}, z^{1}, \ldots z^{n-1}, y^{0}, y^{1}, \ldots\right) .
$$


And that, if $n$ is even, then

$$
w=\left(y^{0}, y^{1}, \ldots y^{n-1}, y^{0}, y^{1}, \ldots\right)
$$

In both cases, $w$ is not eventually constant, as $y_{0} \neq y_{1}$ because $s_{2}^{0} \neq s_{2}^{2}$.

Let $w^{k-1} \neq w^{k}$. If $w^{k-1}=\left(s_{1}^{k}, s_{2}^{k-1}\right)$, and $w^{k}=\left(s_{1}^{k}, s_{2}^{k+1}\right)$, then $s_{2}^{k-1} \neq s_{2}^{k+1}=\beta_{2}\left(s_{1}^{k}\right)$. Best-responses are unique, then $u_{2}\left(s_{1}^{k}, s_{2}^{k-1}\right)<u_{2}\left(s_{1}^{k}, s_{2}^{k+1}\right)$. Similarly if $w^{k}=\left(s_{1}^{k+1}, s_{2}^{k}\right)$, and $w^{k-1}=\left(s_{1}^{k-1}, s_{2}^{k}\right)$. So, $w$ is an infinite improvement path.

By Monderer and Shapley's (1996) Corollary 2.2, $\Gamma$ has at least one equilibrium. If it has two or more equilibria, it is a GSC by Theorem 5. Let $\Gamma$ have a unique equilibrium. Lemma 10 implies that $\Gamma$ cannot have an infinite improvement path, so Lemma 11 implies that it cannot have a best-response cycle; by Theorem 2, then, $\Gamma$ is a GSC.

\section{Proofs}

\subsection{Proof of Theorem 3}

I prove the second statement in Step 1. Steps 2 and 3 prove the first statement when $X$ is finite-Step 2 constructs an order such that $\phi$ is weakly increasing, and Step 3 checks that this is a total order. Steps 4 and 5 prove the first statement for arbitrary $X$ by non-standard analysis methods. The idea is to embed $X$ in a hyperfinite set, apply the result for finite sets to get an order that works in the hyperfinite set, and then restrict the order to $X$.

STEP 1. Let $\leq$ be a total order on $X$ such that $\phi$ is increasing in the strong set order, and let $x \neq e$. Suppose, by way of contradiction, that there is $\left\{x_{m}\right\}_{m=0}^{K} \subseteq X$ such that $x=x_{0}=x_{K}$ and $x_{m} \in \phi\left(x_{m-1}\right), 1 \leq m \leq K-1$. Note that we must have $x_{m} \neq e$ for all $m$ because $\phi(e)=\{e\}$ would imply that $x=e$.

Since $X$ is totally ordered by $\leq$, either $x<x_{K-1}$ or $x_{K-1}<x$, as $x=x_{K-1}$ is ruled out because $x$ is not a fixed point. Suppose that $x<x_{K-1}$, I will show by induction that $x_{K}<x_{K-1}<\ldots<x_{0}=x$, a contradiction. First, $x<x_{K-1}$ implies that $\phi(x) \ni x_{1}$ is smaller than $\phi\left(x_{K-1}\right) \ni x$ in the strong set order, so $x_{1} \wedge x \in \phi(x)$. Now, $x_{1} \wedge x \in\left\{x_{1}, x\right\}$ because $\leq$ is a total order, and $x \notin \phi(x)$ because $x$ is not a fixed point. So we must have $x_{1}<x$. Now for the inductive step, I want to show that, if $x_{m}<x_{m-1}$, then $x_{m+1}<x_{m}$ $(1 \leq m \leq K-1)$. If $x_{m}<x_{m-1}$, then $\phi\left(x_{m}\right) \ni x_{m+1}$ is smaller than $\phi\left(x_{m-1}\right) \ni x_{m}$ in the 
strong set order. Then $\left\{x_{m+1}, x_{m}\right\} \ni x_{m+1} \wedge x_{m} \in \phi\left(x_{m}\right)$, but $x_{m} \notin \phi\left(x_{m}\right)$ as $x_{m} \neq e$, so $x_{m+1}<x_{m}$.

If, instead, $x>x_{K-1}$ we can apply an analogous argument to reach a contradiction. So there cannot be a $K$ with $x \in \phi^{K}(x)$.

Step 2. Let $X$ be finite. Let $\phi: X \rightarrow X$ be a correspondence with a unique fixed point $e \in X$, and no cycles besides $e$. For all $x \in X$ there is at least one sequence $\left\{x_{m}\right\}_{m=0}^{K}$ with $x_{0}=x, x_{m} \in \phi\left(x_{m-1}\right), m=1,2, \ldots K$, and $x_{K}=e$; that is a path connecting $x$ and $e, K$ is the length of the path. To see that such a path must exist, note that, by absence of cycles, if $x_{m} \neq e$ for $m=1,2, \ldots K$, then $\left\{x_{m}\right\}_{m=0}^{K}$ are all distinct, so $X$ has at least $K+1$ elements. $X$ is finite, so we must have, for $K$ large enough, that $x_{K}=e$. For each $x \in X$, let $K_{x}$ be the smallest $K$ such that there is a path of length $\mathrm{K}$ connecting $x$ and $e$. Clearly, $K_{e}=0$. Fix, for each $x$, a path of minimal length $\left\{x_{m}\right\}_{m=0}^{K_{x}}$ connecting $x$ and e. Note that $K_{x_{1}}=K_{x}-1$, as the $K$ 's are minimal.

Define $X_{m}=\left\{x \in X: K_{x}=m\right\}, m=0,1, \ldots M$, where $M$ is such that $X=$ $\cup_{m=0}^{M} X_{m}$. The collection $\left\{X_{m}\right\}_{m=0}^{M}$ is a partition of $X$ because it covers $X$ and has disjoint elements, as the $K_{x} \mathrm{~s}$ are minimal.

I shall define recursively an order $\leq_{m}$ on $X_{m}, m=0,1, \ldots M$. Let $\preceq$ be a total order on $X$ such that $e$ is the largest element of $X$ (for example, embed $X$ in $\mathbf{N}$ such that $e$ is mapped to a number larger than any other element in $X$, and take the relative order on $X)$. Let $\leq_{0}$ on $X_{0}$, be the restriction of $\preceq$ to $X_{0}$. Given a total order $\leq_{m}$ on $X_{m}$, let $\leq_{m+1}$ on $X_{m+1}$ be defined by, $x \leq_{m+1} y$ if $x_{1}<_{m} y_{1}$ or if $x_{1}=y_{1}$ and $x \preceq y$, where $x_{1}$ and $y_{1}$ are the first elements in the paths connecting $x$ and $y$, respectively, to $e$. Note that $\leq_{m}$ is in fact a total order because it is a lexicographic order.

Finally, define a total order $\leq$ on $X$ by $x \leq y$ if either $k_{y}<k_{x}$ or if $k_{y}=k_{x}$ and $x \leq_{k_{x}} y$. Note that $e$ is the largest element in $X$.

STEP 3. I shall check that $\phi$ is weakly increasing. Let $x, y \in X$ and $\left\{x_{m}\right\}_{m=0}^{K_{x}}$, $\left\{y_{m}\right\}_{m=0}^{K_{y}}$ be the paths of minimal length connecting $x$ and $y$ to $e$ from step 2 .

Let $x \leq y$. If $K_{y}<K_{x}$ then there is $x_{1} \in \phi(x)$ and $y_{1} \in \phi(y), x_{1} \in\left\{x_{m}\right\}_{m=0}^{K_{x}}$, $y_{1} \in\left\{y_{m}\right\}_{m=0}^{K_{y}}$, with $K_{y_{1}}=K_{y}-1<K_{x}-1=K_{x_{1}}$ because the $K$ 's are minimal. Then, $x_{1} \leq y_{1}$. If $K_{y}=K_{x}$ then there is $x_{1} \in \phi(x)$ and $y_{1} \in \phi(y), x_{1} \in\left\{x_{m}\right\}_{m=0}^{K_{x}}, y_{1} \in\left\{y_{m}\right\}_{m=0}^{K_{y}}$ with $x_{1} \leq_{K_{x}+1} y_{1}$. Then $K_{y_{1}}=K_{y}-1=K_{x}-1=K_{x_{1}}$, because the $K$ 's are minimal. 
Then, $x_{1} \leq y_{1}$. In both cases there is $x_{1} \in \phi(x)$ and $y_{1} \in \phi(y)$ with $x_{1} \leq y_{1}$, thus proving that $\phi$ is weakly increasing

To prove that $\leq$ is a total order, note first that $x \leq x$ because $x$ is in some $X_{m}$, and $x \leq_{m} x$, as $\leq_{m}$ is an order; thus $\leq$ is reflexive. If $x \leq y$ and $y \leq x$ then we must have $K_{x}=K_{y}$, and then $x=y$ because $\leq_{K_{x}}$ is an order. So, $\leq$ is antisymmetric. Let $x \leq y$ and $y \leq z$. If $K_{y}<K_{x}$ or $K_{z}<K_{y}$ then $K_{z}<K_{x}$ and $x \leq z$. If $K_{x}=K_{y}=K_{z}$ then $x \leq z$ follows from transitivity of $\leq_{K_{x}}$. Thus $\leq$ is transitive. Finally, $\leq$ is total because if $x, y \in X$ then there is $m, n$ such that $x \in X_{m}$ and $y \in X_{n}$. If $m<n$ or $n<m, x$ and $y$ are ordered. If $n=m, x$ and $y$ are ordered because $\leq_{m}$ is a total order.

SteP 4. Suppose first that $\phi(e)=\{e\}$. Let $H$ be a hyperfinite set with $X \subseteq H \subseteq * X$. For all $x \in X, \phi(x)$ is finite, then $* \phi$ takes hyperfinite values, and therefore $* \phi(H)$ is hyperfinite, as it is the hyperfinite union of hyperfinite sets. Let $\tilde{H}=H \cup * \phi(H), \tilde{H}$ is hyperfinite.

By the Transfer Principle, $h \in H$ implies $(h \in * \phi(h) \Rightarrow h=e)$. Define $\tilde{\phi}: \tilde{H} \rightarrow \tilde{H}$ by

$$
\tilde{\phi}(h)= \begin{cases}* \phi(h) & \text { if } h \in H \\ \{e\} & \text { if } h \in \tilde{H} \backslash H .\end{cases}
$$

$\tilde{\phi}$ is internal, and $\tilde{\phi}(e)=* \phi(e)=\{e\}$. I show that $e$ is the only fixed point of $\tilde{\phi}$. Suppose $x \in \tilde{\phi}(x)$. First, if $x \in H$, then $\tilde{\phi}(x)=* \phi(x)$, so $x \in * \phi(x)$ implies that $x=e$ by Transfer. Second, if $x \in \tilde{H} \backslash H$ then $\tilde{\phi}(x)=\{e\}$, so $x=e \in H$, a contradiction.

To show that $\tilde{\phi}$ has no cycles except for $e$, suppose that $x \in \tilde{\phi}^{K}(x)$ for some $x \in \tilde{H}$ and $K \in * \mathbf{N}$. Note that $x \in H$ because $x \in \tilde{H} \backslash H$ implies that $\tilde{\phi}^{m}(x)=\{e\}$ for all $m$, and $e \in H$. There is a sequence $\left\{x_{m}\right\}_{m=0}^{K}$ with $x_{0}=x_{K}=x$ and $x_{m} \in \tilde{\phi}\left(x_{m-1}\right)$, $m=1,2, \ldots K$. If there is $m$ such that $x_{m} \in \tilde{H} \backslash H$ then $e=x_{l}, m \leq l \leq K$, so $x=e$. On the other hand, if $x_{m-1} \in H$ for all $m$, then $x_{m} \in \tilde{\phi}\left(x_{m-1}\right)=* \phi\left(x_{m-1}\right)$ for all $m$. Then $x$ is a cycle of $* \phi$, so $x=e$ by Transfer.

Then, $\tilde{H}$ is hyperfinite, $\tilde{\phi}: \tilde{H} \rightarrow \tilde{H}$ has a unique fixed point $e$, and no cycles but $e$. By steps 2 and 3 above and the Transfer Principle, there is a total order $\triangleleft$ on $H$ such that $x \triangleleft e$ for all $x \in H$, and such that $x \triangleleft y$ implies that there is $z \in \tilde{\phi}(x)$ and $z^{\prime} \in \tilde{\phi}(y)$ with $z \triangleleft z^{\prime}$. Let $\leq$ be the restriction of $\triangleleft$ to $X \subseteq \tilde{H}, \leq$ is a total order on $X$, and $e$ is its largest element. 
To verify that $\phi$ is weakly increasing, let $x \leq y$. Then, $x \triangleleft y$ implies that there is $z \in \tilde{\phi}(x)$ and $z^{\prime} \in \tilde{\phi}(y)$ with $z \triangleleft z^{\prime}$. But $\tilde{\phi}(x)=* \phi(x)=\phi(x)$ and $\tilde{\phi}(y)=* \phi(y)=\phi(y)$, as $x, y \in X \subseteq H$. Then $z \in \phi(x), z^{\prime} \in \phi(y)$, and $z \leq z^{\prime}$ because $z \triangleleft z^{\prime}$.

Step 5. Finally, let $\phi(e) \neq\{e\}$, and let $\phi^{\prime}: X \rightarrow X$ coincide with $\phi$ on $X \backslash\{e\}$, and take the value $\{e\}$ on e. By step 4 there is an order $\leq$ on $X$ such that $e$ is its largest element and such that $\phi^{\prime}$ is weakly increasing. Now we check that $\phi$ is weakly increasing in this order as well. We only need to prove that if $x \in X$ then there is $z \in \phi(x)$ and $z^{\prime} \in \phi(e)$ with $z \leq z^{\prime}$. But, since $e \in \phi(e)$ and $e$ is the largest element in $X$, we can set $z^{\prime}=e$ and be done.

\subsection{Proof of Theorem 7}

First I construct an order $\leq$ on $X$, then I show that $\leq$ satisfies the statements in the theorem.

Denote by $\mathbb{C}$ the set of cycles of $f$, so

$$
\mathbb{C}=\left\{x \in X: \exists n \in \mathbf{N} \text { s.t. } f^{n}(x)=x\right\} \text {. }
$$

Note that $\underline{e}, \bar{e} \in \mathbb{C}$.

A path in $X$ is a sequence $\pi=\left\{x_{1}, \ldots x_{L}\right\}$ such that, $x_{l} \notin \mathbb{C}$ for $1 \leq l \leq L$, $f\left(x_{1}\right) \in \mathbb{C} \backslash\{\underline{e}, \bar{e}\}, f^{-1}\left(x_{L}\right)=\emptyset$, and $x_{l}=f\left(x_{l+1}\right)$ for $1 \leq l \leq L-1$. Let $\Pi$ be the set of all paths. Note that $\Pi$ is finite.

If $\pi=\left\{x_{1}, \ldots x_{L}\right\}$ is a path, let $H_{\pi}=L$ denote the length of $\pi$. For each $x_{l} \in \pi$, let $h_{\pi}\left(x_{l}\right)=l$. Let $\mathbb{A}=\cup\{\pi: \pi \in \Pi\}$.

If $e \in X$ is a fixed point of $f$, the basin of $e$, denoted $\mathbb{B}_{e}$, is the set of points $z \in X$ such that $e=f^{n}(z)$ for some $n$. For each $z \in \mathbb{B}_{e}$, let $g_{e}(z)=\inf \left\{n \in \mathbf{N}: f^{n}(z)=e\right\}$. Define $g_{e}(e)=0$. Let $H_{e}=\sup \left\{g_{e}(z): z \in \mathbb{B}_{e}\right\}$.

Suppose, without loss of generality, that $H_{\underline{e}} \geq H_{\bar{e}}$ (if $H_{\underline{e}}<H_{\bar{e}}$ just relabel $\underline{e}$ and $\bar{e}$ ). For each $z \in \mathbb{A}$, let

$$
g(z)=\inf \left\{H_{\underline{e}}-\left[H_{\pi}-h_{\pi}(z)\right]: \pi \in \Pi \text { and } z \in \pi\right\} .
$$

I claim that $X=\mathbb{C} \cup \mathbb{A} \cup \mathbb{B}_{\underline{e}} \cup \mathbb{B}_{\bar{e}}$ : Let $z \in X$. Since $X$ is finite, for large enough $n$, either $f^{n}(z)$ is a fixed point or it is a cycle. If $f^{n}(z)$ is eventually $\underline{e}$ or $\bar{e}$, then $x \in\left(\mathbb{B}_{\underline{e}} \cup \mathbb{B}_{\bar{e}}\right)$. 
If $f^{n}(z)$ is eventually some other $x \in \mathbb{C}$, then either $z \in \mathbb{C}$ in the first place, or $z \in \mathbb{A}$. In any case, $x \in \mathbb{C} \cup \mathbb{A} \cup \mathbb{B}_{\underline{e}} \cup \mathbb{B}_{\bar{e}}$, which establishes the claim. Also note that, by definition of $\mathbb{C}, \mathbb{A}, \mathbb{B}_{\underline{e}}$ and $\mathbb{B}_{\bar{e}}, \mathbb{A} \cap \mathbb{C}=\emptyset, \mathbb{A} \cap \mathbb{B}_{\underline{e}}=\emptyset, \mathbb{A} \cap \mathbb{B}_{\bar{e}}=\emptyset, \mathbb{B}_{\underline{e}} \cap \mathbb{B}_{\bar{e}}=\emptyset, \mathbb{B}_{\underline{e}} \cap \mathbb{C}=\{\underline{e}\}$ and $\mathbb{B}_{\bar{e}} \cap \mathbb{C}=\{\bar{e}\}$.

Before I define $\leq$ I need two auxiliary orders. I shall define $\leq_{\underline{e}}$ on $\mathbb{B}_{\underline{e}}$ and $\leq_{\bar{e}}$ on $\mathbb{B}_{\bar{e}}$ such that $f$ restricted to either $\mathbb{B}_{\underline{e}}$ or $\mathbb{B}_{\bar{e}}$ is monotone increasing, and such that $\underline{e}$ is the largest element of $\mathbb{B}_{\underline{e}}$, and $\bar{e}$ is the smallest element of $\mathbb{B}_{\bar{e}}$. First, I claim that $f\left(\mathbb{B}_{\underline{e}}\right) \subseteq \mathbb{B}_{\underline{e}}$, and that $f\left(\mathbb{B}_{\bar{e}}\right) \subseteq \mathbb{B}_{\bar{e}}$. To see this, let $x \in \mathbb{B}_{\underline{e}}$. If $f(\underline{e})=\underline{e}$, then $f(x) \in \mathbb{B}_{\underline{e}}$. Suppose that $f(\underline{e}) \neq \underline{e}$, then there is $n \in \mathbf{N}$ such that $f^{n}(x)=\underline{e}$. But $f(x) \neq \underline{e}$ implies $n \geq 2$, so $f^{n-1}(f(x))=\underline{e}$, and $f(x) \in \mathbb{B}_{\underline{e}}$. The proof that $f\left(\mathbb{B}_{\bar{e}}\right) \subseteq \mathbb{B}_{\bar{e}}$ is similar.

Now I can obtain $\leq_{\underline{e}}$ and $\leq_{\bar{e}}$ from Theorem 2. First, $\left.f\right|_{\mathbb{B}_{\underline{e}}}$, the restriction of $f$ to $\mathbb{B}_{\underline{e}}$, maps $\mathbb{B}_{\underline{e}}$ into $\mathbb{B}_{\underline{e}}$, has exactly one fixed point, and no cycles. By Theorem 2 , there is a total order - say $\leq_{\underline{e}}$ - on $\mathbb{B}_{\underline{e}}$ such that $\left.f\right|_{\mathbb{B}_{\underline{e}}}$ is monotone increasing and $\underline{e}$ is the largest element of $\mathbb{B}_{\underline{e}}$. Second, by a similar argument, there is a total order $\leq_{\bar{e}}$ on $\mathbb{B}_{\bar{e}}$ such that $\left.f\right|_{\mathbb{B}_{\bar{e}}}$ is monotone increasing and $\bar{e}$ is the smallest element of $\mathbb{B}_{\bar{e}}$ - simply reverse the order that Theorem 2 delivers on $\mathbb{B}_{\bar{e}}$.

Let $\underline{x}=\inf _{\leq \underline{e}} \mathbb{B}_{\underline{e}}$ and $\bar{x}=\sup _{\leq \bar{e}} \mathbb{B}_{\bar{e}}$

Definition 12 Define the order $\leq$ on $X$ by: $z \leq z^{\prime}$ if and only if one (or more) of the following is true:

1. $z=z^{\prime}$,

2. $z, z^{\prime} \in \mathbb{B}_{\underline{e}}$ and $z \leq_{\underline{e}} z^{\prime}$ or $z, z^{\prime} \in \mathbb{B}_{\bar{e}}$ and $z \leq_{\bar{e}} z^{\prime}$,

3. $z \in \mathbb{B}_{\underline{e}}$ and $z^{\prime} \in \mathbb{C}$,

4. $z^{\prime} \in \mathbb{B}_{\bar{e}}$ and $z \notin \mathbb{B}_{\bar{e}}$,

5. $z \in \mathbb{B}_{\underline{e}}, z^{\prime} \in \mathbb{A}$, and $g_{\underline{e}}(z) \geq g\left(z^{\prime}\right)$.

\section{Remark.}

1. Note that $\leq$ coincides with $\leq_{\underline{e}}$ on $\mathbb{B}_{\underline{e}}$ and with $\leq_{\bar{e}}$ on $\mathbb{B}_{\bar{e}}$.

2. Since $\leq$ coincides with $\leq_{\underline{e}}$ on $\mathbb{B}_{\underline{e}}, g_{\underline{e}}$ is monotone decreasing: $z<z^{\prime}$ implies $g_{\underline{e}}(z) \geq$ $g_{\underline{e}}\left(z^{\prime}\right)$. Further, by construction of $\leq_{\underline{e}}, g_{\underline{e}}(z)>g_{\underline{e}}\left(z^{\prime}\right)$ implies that $z<z^{\prime}$. 
3. If $z \in \mathbb{B}_{\underline{e}}$ and $z<\underline{e}$, then $g_{\underline{e}}(f(z))=g_{\underline{e}}(z)-1$.

4. For all $z \in X, \underline{x} \leq z$, so $\underline{x}$ is the smallest element in $X$.

I now prove that $(X, \leq)$ is a complete lattice (steps 1 and 2 ), that $f$ is monotone increasing (step 3), and that any other lattice order that makes $f$ monotone increasing must have a larger interval prediction (step 4).

STEP 1: I prove that $\leq$ is a partial order. By definition of $\leq$ (item 1$), \leq$ is reflexive. I need to prove that it is antisymmetric and transitive.

To prove that $\leq$ is antisymmetric, let $z \leq z^{\prime}$ and $z \neq z^{\prime}$, I shall prove that $z^{\prime} \not z$. First, $z \leq z^{\prime}$ cannot be due to item 1 of the definition of $\leq$. Second, if $z \leq z^{\prime}$ because of item 2 , then $z^{\prime} \not \leq z$, as $\leq_{\underline{e}}$ and $\leq_{\bar{e}}$ are antisymmetric. Third, if $z \in B_{\underline{e}}$ then $z^{\prime} \leq z$ only if $z^{\prime} \in \mathbb{B}_{\underline{e}}$ as well. So, if $z \leq z^{\prime}$ by item 3 or item 5 we get immediately $z^{\prime} \not \leq z$. Fourth, if $z^{\prime} \in \mathbb{B}_{\bar{e}}$ then $z^{\prime} \leq z$ only if $z \in \mathbb{B}_{\bar{e}}$ as well. So, if $z \leq z^{\prime}$ by item 4 we get immediately $z^{\prime} \not z$.

Now I shall prove that $\leq$ is transitive: let $z \leq z^{\prime}$ and $z^{\prime} \leq z^{\prime \prime}$, I shall prove that $z \leq z^{\prime \prime}$. If either $z \leq z^{\prime}$ or $z^{\prime} \leq z^{\prime \prime}$ is due to item 1 , it is immediate that $z \leq z^{\prime \prime}$. So, suppose that $z \neq z^{\prime}$ and $z^{\prime} \neq z^{\prime \prime}$.

First, let $z \leq z^{\prime}$ be due to item 2. If $z, z^{\prime} \in \mathbb{B}_{\bar{e}}$ then it must be that $z^{\prime \prime} \in \mathbb{B}_{\bar{e}}$, but then $z \leq z^{\prime \prime}$, as $\leq_{\bar{e}}$ is transitive. Now let $z, z^{\prime} \in \mathbb{B}_{\underline{\underline{e}}}$. Assume $z^{\prime \prime} \in \mathbb{B}_{\underline{e}} \cup \mathbb{B}_{\bar{e}}$. Either $z^{\prime \prime} \in \mathbb{B}_{\bar{e}}$ so $z \leq z^{\prime \prime}$ by item 4 or $z^{\prime \prime} \in \mathbb{B}_{\underline{e}}$ and $z \leq z^{\prime \prime}$ because $\leq_{\underline{e}}$ is transitive. Now assume that $z^{\prime \prime} \notin \mathbb{B}_{\underline{e}} \cup \mathbb{B}_{\bar{e}}$. Either $z^{\prime \prime} \in \mathbb{C}$ and we get $z \leq z^{\prime \prime}$ by item 3 ; or $z^{\prime \prime} \in \mathbb{A}$, but then $z^{\prime} \leq z^{\prime \prime}$ is due to item 5 , so $z \leq_{\underline{e}} z^{\prime}$ implies that $g_{\underline{e}}(z) \geq g_{\underline{e}}\left(z^{\prime}\right) \geq g\left(z^{\prime \prime}\right)$. Thus $z \leq z^{\prime \prime}$ by item 5 .

Second, let $z \leq z^{\prime}$ be due to item $3 ; z^{\prime} \leq z^{\prime \prime}$ can then only be due to item 4 . So $z^{\prime \prime} \in \mathbb{B}_{\bar{e}}$, and we have $z \leq z^{\prime \prime}$ by item 4 .

Third, if $z \leq z^{\prime}$ is due to item 4 , then $z^{\prime} \in \mathbb{B}_{\bar{e}}$, so $z^{\prime} \leq z^{\prime \prime}$ implies that $z^{\prime \prime} \in \mathbb{B}_{\bar{e}}$, and we have $z \leq z^{\prime \prime}$ by item 4 .

Fourth, if $z \leq z^{\prime}$ is due to item 5 , then $z^{\prime} \in \mathbb{A}$. But then $z^{\prime} \leq z^{\prime \prime}$ implies that $z^{\prime \prime} \in \mathbb{B}_{\bar{e}}$, so we get $z \leq z^{\prime \prime}$ by item 4 .

SteP 2: I prove that $(X, \leq)$ is a lattice. Since $X$ is finite, $(X, \leq)$ is then a complete lattice. 
Let $z, z^{\prime} \in X$. If $z \leq z^{\prime}$ or $z^{\prime} \leq z$, then $z \vee z^{\prime}$ and $z \wedge z^{\prime}$ are trivial. So let $z$ and $z^{\prime}$ be unordered, and note that, when $z$ and $z^{\prime}$ are unordered, $z \vee z^{\prime}$ is the smallest element of $\{\tilde{z} \in X: z<\tilde{z}\} \cap\left\{\tilde{z} \in X: z^{\prime}<\tilde{z}\right\}$, and $z \wedge z^{\prime}$ is the largest element of $\{\tilde{z} \in X: \tilde{z}<z\} \cap$ $\left\{\tilde{z} \in X: \tilde{z}<z^{\prime}\right\}$.

I shall need the following two facts, the facts are immediate from the definition of $\leq$.

FACT 1: If $z \in(\mathbb{C} \cup \mathbb{A}) \backslash\{\bar{e}\}$, then $\underline{x} \in\{\tilde{z} \in X: \tilde{z}<z\} \subseteq \mathbb{B}_{\underline{e}}$.

FACT 2: If $z \in(\mathbb{C} \cup \mathbb{A}) \backslash\{\bar{e}\}$, then $\{\tilde{z} \in X: z<\tilde{z}\}=\mathbb{B}_{\bar{e}}$.

First, let $z \in \mathbb{B}_{\underline{e}}$. Since $z$ and $z^{\prime}$ are unordered, we must have $z^{\prime} \in \mathbb{A}$. Also, $z \neq \underline{x}$, as $\underline{x}$ is the smallest element in $X$, so $z=\underline{x}$ would imply that $z$ and $z^{\prime}$ are ordered. Note that $z \in \mathbb{B}_{\underline{e}} \backslash\{\underline{x}\}$ implies that $\underline{x} \in\{\tilde{z} \in X: \tilde{z}<z\} \subseteq \mathbb{B}_{\underline{e}}$, and $\{\tilde{z} \in X: z<\tilde{z}\}=\mathbb{B}_{\bar{e}}$. Hence, by facts 1 and 2 ,

$$
\underline{x} \in\{\tilde{z} \in X: \tilde{z}<z\} \cap\left\{\tilde{z} \in X: \tilde{z}<z^{\prime}\right\} \subseteq \mathbb{B}_{\underline{e}}
$$

and

$$
\{\tilde{z} \in X: z<\tilde{z}\} \cap\left\{\tilde{z} \in X: z^{\prime}<\tilde{z}\right\}=\mathbb{B}_{\bar{e}}
$$

But $\leq$ is a total order on $\mathbb{B}_{\underline{e}}$, and $\mathbb{B}_{\underline{e}}$ is finite, so any non-empty subset of $\mathbb{B}_{\underline{e}}$ has a largest element. Thus $z \wedge z^{\prime}$ exists. Similarly, $\mathbb{B}_{\bar{e}}$ is finite and totally ordered by $\leq$. So $\mathbb{B}_{\bar{e}}$ has a smallest element (namely $\bar{e}$ ), and $z \vee z^{\prime}$ exists.

Second, let $z \in \mathbb{A}$. Since $z$ and $z^{\prime}$ are unordered we must have $z^{\prime} \notin \mathbb{B}_{\bar{e}}$. Further, if $z^{\prime} \in \mathbb{B}_{\underline{e}}$ then we already proved above that $z \wedge z^{\prime}$ and $z \vee z^{\prime}$ exist. Let $z^{\prime} \in(\mathbb{C} \cup \mathbb{A}) \backslash\{\bar{e}\}$. Using facts 1 and 2 in as above, we know that $\{\tilde{z} \in X: \tilde{z}<z\} \cap\left\{\tilde{z} \in X: \tilde{z}<z^{\prime}\right\}$ is a non-empty subset of $\mathbb{B}_{\underline{e}}$, and that $\{\tilde{z} \in X: z<\tilde{z}\} \cap\left\{\tilde{z} \in X: z^{\prime}<\tilde{z}\right\}=\mathbb{B}_{\bar{e}}$. Since $\mathbb{B}_{\underline{e}}$ and $\mathbb{B}_{\bar{e}}$ are finite, totally ordered sets, $z \wedge z^{\prime}$ and $z \vee z^{\prime}$ exist in $X$.

Third, let $z \in \mathbb{C} \backslash\{\underline{e}, \bar{e}\}$. Since $z$ and $z^{\prime}$ are unordered, we must have $z^{\prime} \in(\mathbb{C} \cup \mathbb{A}) \backslash\{\underline{e}, \bar{e}\}$. If $z^{\prime} \in \mathbb{A}$, then we know that $z \wedge z^{\prime}$ and $z \vee z^{\prime}$ exist in $X$ from the argument in the previous paragraph. If, $z^{\prime} \in \mathbb{C}$, then

$$
\underline{x} \in\{\tilde{z} \in X: \tilde{z}<z\}=\left\{\tilde{z} \in X: \tilde{z}<z^{\prime}\right\}=\mathbb{B}_{\underline{e}}
$$

and

$$
\{\tilde{z} \in X: z<\tilde{z}\}=\left\{\tilde{z} \in X: z^{\prime}<\tilde{z}\right\}=\mathbb{B}_{\bar{e}} .
$$

Again $\mathbb{B}_{\underline{e}}$ and $\mathbb{B}_{\bar{e}}$ are finite and totally ordered by $\leq$, so $z \wedge z^{\prime}$ and $z \vee z^{\prime}$ exist in $X$. 
Finally, if $z \in \mathbb{B}_{\bar{e}}, z$ and $z^{\prime}$ cannot be unordered, as $z^{\prime} \notin \mathbb{B}_{\bar{e}}$ implies $z^{\prime} \leq z$, and $z^{\prime} \in \mathbb{B}_{\bar{e}}$ implies that $z$ and $z^{\prime}$ are ordered, as $\mathbb{B}_{\bar{e}}$ is totally ordered.

Step 3: Now I prove that $f$ is monotone increasing.

Let $z \leq z^{\prime}$. I need to prove that $f(z) \leq f\left(z^{\prime}\right)$. First, if $z \leq z^{\prime}$ is due to item 1 , then $f(z) \leq f\left(z^{\prime}\right)$ because $f(z)=f\left(z^{\prime}\right)$. And if $z \leq z^{\prime}$ is due to item 2 it is immediate that $f(z) \leq f\left(z^{\prime}\right)$ because $\leq$ concides with $\leq_{\underline{e}}$ and $\leq_{\bar{e}}$ on $\mathbb{B}_{\underline{e}}$ and $\mathbb{B}_{\bar{e}}$, and $\leq_{\underline{e}}$ and $\leq_{\bar{e}}$ are constructed so that $f$ is monotone increasing.

Second, let $z \leq z^{\prime}$ be due to item 3. $z \in \mathbb{B}_{\underline{e}}$ implies that $f(z) \in \mathbb{B}_{\underline{e}}$, and $z^{\prime} \in \mathbb{C}$ implies that $f\left(z^{\prime}\right) \in \mathbb{C}$. So, we get $f(z) \leq f\left(z^{\prime}\right)$ by item 3 .

Third, let $z \leq z^{\prime}$ be due to item $4 . z^{\prime} \in \mathbb{B}_{\bar{e}}$ implies that $f\left(z^{\prime}\right) \in \mathbb{B}_{\bar{e}}$. Also, $z \notin \mathbb{B}_{\bar{e}}$ implies $f(z) \notin \mathbb{B}_{\bar{e}}$, as, for any $\tilde{z} \in X, f(\tilde{z}) \in \mathbb{B}_{\bar{e}}$ implies $\tilde{z} \in \mathbb{B}_{\bar{e}}$ by the definition of $\mathbb{B}_{\bar{e}}$. Hence $f(z) \leq f\left(z^{\prime}\right)$ due to item 4 .

Fourth, let $z \leq z^{\prime}$ be due to item 5. So, $z \in \mathbb{B}_{\underline{e}}$ and $z^{\prime} \in \mathbb{A}$. If $f\left(z^{\prime}\right) \in \mathbb{C}$, then $f(z) \leq f\left(z^{\prime}\right)$ by item 3 , as $f(z) \in \mathbb{B}_{\underline{e}}$. Assume $f\left(z^{\prime}\right) \notin \mathbb{C}$. Since $z \leq z^{\prime}, g_{\underline{e}}(z) \geq g\left(z^{\prime}\right)$. Now, $\Pi$ is finite, so there is a path $\hat{\pi}$ such that $g\left(z^{\prime}\right)=H_{\underline{e}}-\left[H_{\hat{\pi}}-h_{\hat{\pi}\left(z^{\prime}\right)}\right]$. Since $f\left(z^{\prime}\right) \notin \mathbb{C}$, we must have $f\left(z^{\prime}\right) \in \hat{\pi}$, and $h_{\hat{\pi}}\left(f\left(z^{\prime}\right)\right)=h_{\hat{\pi}}\left(z^{\prime}\right)-1$. So,

$$
\begin{aligned}
g\left(f\left(z^{\prime}\right)\right) & \leq H_{\underline{e}}-\left[H_{\hat{\pi}}-h_{\hat{\pi}}\left(f\left(z^{\prime}\right)\right)\right] \\
& =H_{\underline{e}}-\left[H_{\hat{\pi}}-h_{\hat{\pi}}\left(z^{\prime}\right)\right]-1 \\
& =g\left(z^{\prime}\right)-1 .
\end{aligned}
$$

On the other hand, $z \in \mathbb{B}_{\underline{e}}$, so either $z=\underline{e}$ or $z<\underline{e}$. First, if $z=\underline{e}$, then $f(z)=\underline{e}$, so $g_{\underline{e}}(z)=g_{\underline{e}}(f(z))$. Then $g_{\underline{e}}(z) \geq g\left(z^{\prime}\right)$ and $g\left(f\left(z^{\prime}\right)\right) \leq g\left(z^{\prime}\right)-1$ implies that $g_{\underline{e}}(f(z)) \geq$ $g\left(f\left(z^{\prime}\right)\right)$, so $f(z) \leq f\left(z^{\prime}\right)$ by item 5 .

Second, if $z<\underline{e}$, then $g_{\underline{e}}(f(z))=g_{\underline{e}}(z)-1$. So, $g_{\underline{e}}(z) \geq g\left(z^{\prime}\right)$ implies that $g_{\underline{e}}(f(z))=$ $g_{\underline{e}}(z)-1 \geq g\left(z^{\prime}\right)-1 \geq g\left(f\left(z^{\prime}\right)\right)$. Thus $f(z) \leq f\left(z^{\prime}\right)$ by item 5 .

STEP 4: Let $\preceq$ be a partial order on $X$, such that $(X, \preceq)$ is a complete lattice, $f$ is monotone increasing, $\underline{e}$ is the smallest fixed point of $f$, and $\bar{e}$ is the largest fixed point of $f$. Let $z \in X$ be such that $\underline{e} \leq z \leq \bar{e}$. I prove that $\underline{e} \preceq z \preceq \bar{e}$.

Let $x^{*}=\inf _{\preceq} X$. By Theorem 4.3.4 in Topkis (1998), there is a $k \in \mathbf{N}$ such that $\underline{e}=f^{k}\left(x^{*}\right)$. Let $K$ be the smallest such $k$, and note that $\underline{e}=f^{k}\left(x^{*}\right)$ for all $k \geq K$, as $\underline{e}$ 
is a fixed point of $f$. Note also that the existence of $K$ implies that $x^{*} \in \mathbb{B}_{\underline{e}}$, and note that $H_{\underline{e}} \geq K$.

Now, by the definition of $\leq, \underline{e} \leq z \leq \bar{e}$ implies that either $z \in \mathbb{C}$ or $z \in \mathbb{A}$. Suppose, first, that $z \in \mathbb{C}$. Let $n \in \mathbf{N}$ be such that $z=f^{n}(z)$, at least one such $n$ exists because $z \in \mathbb{C}$. Let $M \in \mathbf{N}$ be such that $K \leq n M$, note that $f^{n M}(z)=z$. Now, $x^{*} \preceq z$, because $x^{*}=\inf _{\preceq} X$. So, $f$ monotone increasing implies that $f^{n M}\left(x^{*}\right) \preceq f^{n M}(z)$. But $K \leq n M$ implies that $f^{n M}\left(x^{*}\right)=\underline{e}$, so we have that $\underline{e} \preceq z$. Repeat the argument with $\sup _{\preceq} X$ in the place of $x^{*}$ and we get $z \preceq \bar{e}$.

Suppose now that $z \in \mathbb{A}$. Then $\underline{e} \leq z$ implies that $g_{\underline{e}}(\underline{e}) \geq g(z)$. But $g_{\underline{e}}(\underline{e})=0$, so $0 \geq g(z)$. There is $\hat{\pi} \in \Pi$ such that $z \in \hat{\pi}$ and $g(z)=H_{\underline{e}}-\left[H_{\hat{\pi}}-h_{\hat{\pi}}(z)\right]$. Thus we have

$$
H_{\hat{\pi}}-h_{\hat{\pi}}(z) \geq H_{\underline{e}} \geq K
$$

Let $z^{\prime}=z_{H_{\hat{\pi}}}$. Then $z^{\prime} \in \pi$, and

$$
f^{H_{\hat{\pi}}-h_{\hat{\pi}}(z)}\left(z^{\prime}\right)=z
$$

Since $x^{*}=\inf _{\preceq} X$, we have $x^{*} \preceq z^{\prime}$, so $f$ monotone increasing and $H_{\hat{\pi}}-h_{\hat{\pi}}(z) \geq K$ implies that

$$
\underline{e}=f^{H_{\hat{\pi}}-h_{\hat{\pi}}(z)}\left(x^{*}\right) \preceq f^{H_{\hat{\pi}}-h_{\hat{\pi}}(z)}\left(z^{\prime}\right)=z .
$$

This proves that $\underline{e} \preceq z$. Now for $\bar{e}$ : Recall that $H_{\underline{e}} \geq H_{\bar{e}}$, so $H_{\hat{\pi}}-h_{\hat{\pi}}(z) \geq H_{\bar{e}}$. By definition of $H_{\bar{e}}$, then,

$$
f^{H_{\hat{\pi}}-h_{\hat{\pi}}(z)}\left(\sup _{\preceq} X\right)=\bar{e} .
$$

But $z^{\prime} \preceq \sup _{\preceq} X$ so

$$
z=f^{H_{\hat{\pi}}-h_{\hat{\pi}}(z)}\left(z^{\prime}\right) \preceq f^{H_{\hat{\pi}}-h_{\hat{\pi}}(z)}\left(\sup _{\preceq} X\right)=\bar{e} .
$$

This proves that $\underline{e} \preceq z \preceq \bar{e}$.

\section{References}

Amir, R. (1996): "Cournot Oligopoly and the Theory of Supermodular Games," Games and Economic Behavior, 15, 132-148.

Amir, R., And V. E. Lambson (2000): "On the Effects of Entry in Cournot Markets," The Review of Economic Studies, 67(2), 235-254. 
Echenique, F. (2000): "Mixed Equilibria in Games of Strategic Complements," Forthcoming in Economic Theory.

(2001): "The Equilibrium Set of a Two-Player Game with Complementarities is a Sublattice.," Forthcoming in Economic Theory.

- (2002): "Comparative Statics by Adaptive Dynamics and The Correspondence Principle," Econometrica, 70(2), 833-844.

Echenique, F., and T. Sabarwal (2000): "Strong Comparative Statics of Equilibrium," Forthcoming in Games and Economic Behavior.

Gabay, D., And H. Moulin (1980): "On the Uniqueness and Stability of Nashequilibria in Noncooperative Games," in Applied Stochastic Control in Econometrics and Management Science, ed. by P. K. A. Bensoussan, and C. Tapiero, pp. 271-293. North Holland Publishing Company.

Lippman, S. A., J. W. Mamer, and K. F. MCCardle (1987): "Comparative Statics in Non-cooperative Games via Transfinitely Iterated Play," Journal of Economic Theory, 41(2), 288-303.

Milgrom, P., And J. Roberts (1990): "Rationalizability, Learning and Equilibrium in Games with Strategic Complementarities," Econometrica, 58(6), 1255-1277.

(1994): "Comparing Equilibria," American Economic Review, 84(3), 441-459.

Milgrom, P., And C. Shannon (1994): "Monotone Comparative Statics," Econometrica, 62(1), 157-180.

Monderer, D., And L. S. Shapley (1996): "Potential Games," Games and Economic Behavior, 14, 124-143.

Morris, S., AND H. S. Shin (2000): "Global Games: Theory and Applications," Prepared for the Eighth World Congress of the Econometric Society.

Sobel, M. J. (1988): "Isotone Comparative Statics in Supermodular Games," mimeo, SUNY at Stony Brooks.

Topkis, D. M. (1979): "Equilibrium Points in Nonzero-Sum n-Person Submodular Games," SIAM Journal of Control and Optimization, 17(6), 773-787.

(1998): Supermodularity and Complementarity. Princeton University Press. 
Villas-Boas, J. M. (1997): "Comparative Statics of Fixed Points," Journal of Economic Theory, 73(1), 183-198.

Vives, X. (1985): "Nash Equilibrium in Oligopoly Games with Monotone Best Responses," CARESS Working Paper 85-10, University of Pennsilvania.

— (1990): "Nash Equilibrium with Strategic Complementarities," Journal of Mathematical Economics, 19(3), 305-321.

Zhou, L. (1994): "The Set of Nash Equilibria of a Supermodular Game Is a Complete Lattice," Games and Economic Behavior, 7(2), 295-300. 